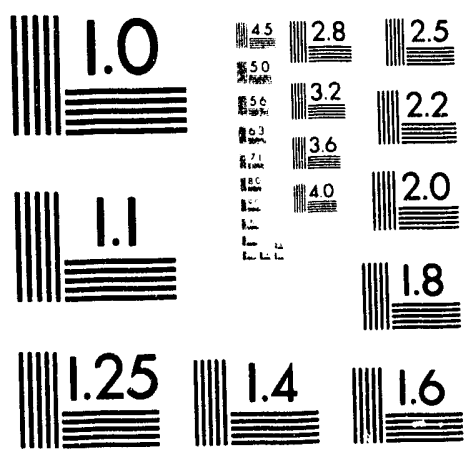



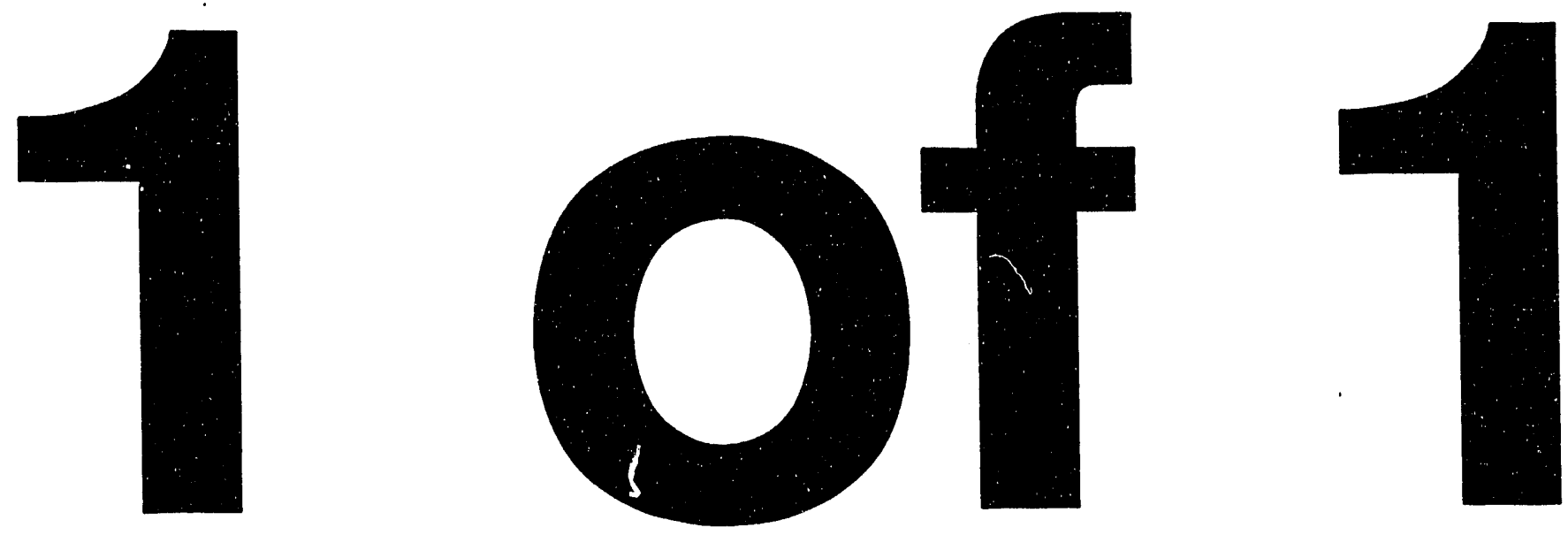


\title{
Optical Diagnostic Instrument for Monitoring Etch Uniformity During Plasma Etching of Polysilicon in a Chlorine-Helium Plasma
}

\author{
Willard A. Hareland \\ Chemical and X-Ray Analysis Department \\ Richard J. Buss \\ Properties of Organic Materials Department \\ Sandia National Laboratories \\ Albuquerque, NM 87185-5800
}

\begin{abstract}
Nonuniform etching is a serious problem in plasma processing of semiconductor materials and has important consequences in the quality and yield of microelectconic components. In many plasmas, etching occurs at a faster rate near the periphery of the wafer, resulting in nonuniform removal of specific materials over the wafer surface. This research was to investigate in situ optical diagnostic techniques for monitoring etch uniformity during plasma processing of microelectronic components. We measured 2-D images of atomic chlorine at $726 \mathrm{~nm}$ in a chlorine-helium plasma during plasma etching of polysilicon in a parallel-plate plasma etching reactor. The 3-D distribution of atomic chlorine was determined by Abel inversion of the plasma image. The experimental results showed that the chlorine atomic emission intensity is at a maximum near the outer radius of the plasma and decreases toward the center. Likewise, the actual etch rate, as determined by profilometry on the processed wafer, was approximately 20 " greater near the edge of the wafer than at its center. There was a direct correlation between the atomic chlorine emission intensity and the etch rate of polysilicon over the wafer surface. Based on these analyses, 3-D imaging would be a useful diagnostic technique for in situ monitoring of etch uniformity on wafers.
\end{abstract}




\section{Acknowledgments}

The authors thank Alex Pimentel for his assistance in fabricating the plasma imaging camera and Pam Ward for her help in the operation of the plasma reactor. 


\section{Contents}

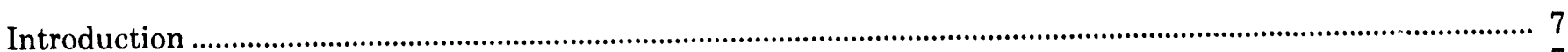

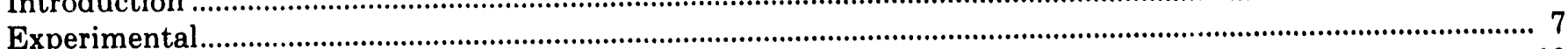

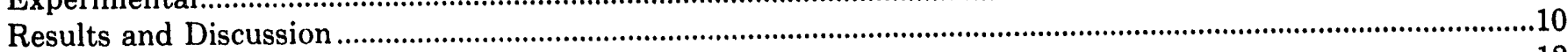

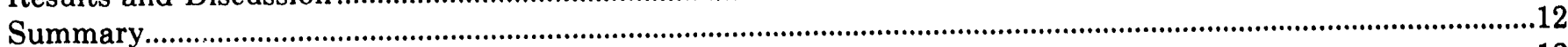

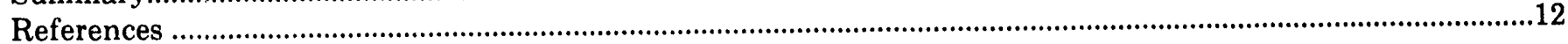

\section{Figures}

1. Imaging Camera for Measuring and Processing Monochromatic Video Images of Radio-Frequency Plasmas

2. Diagram Showing the Relationship Between the Lateral Emission Intensity, I(y), and Radial

Emission Intensity, I(r), Through a Horizontal Plane in an Axisymmetric Plasma

3. Patterned Silicon Wafer (150 mm diameter) with Exposed Silicon Dioxide, Polysilicon, and Photoresist

4. Silicon Wafer in Figure 3 after Etching in a Chlorine-Helium Plasma and Post-Plasma Treatment in an Oxygen Plasma

5. Etch Rate of Polysilicon as a Function of Radial Position on the Plasma-Processed Wafer ............................11

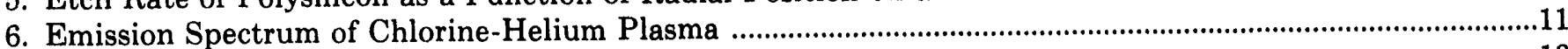

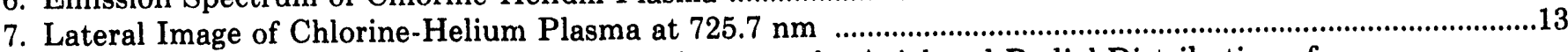

8. Abel Inversion of Lateral Image in Figure 7 Showing the Axial and Radial Distribution of Excited Atomic Chlorine in Chlorine-Helium Plasma.

9. Radial Emission Intensity of Excited Atomic Chlorine Above the Wafer Surface as a Function of Radial Position in Chlorine-Helium Plasma 


\section{Optical Diagnostic Instrument for Monitoring Etch Uniformity During Plasma Etching of Polysilicon in a Chlorine-Helium Plasma}

\section{Introduction}

Glow discharges are used extensively in microelectronic fabrication for etching and thin-film deposition. These low-temperature plasmas produce highly reactive atomic, molecular, and ionized species that react selectively with materials on wafer surfaces. In some processes, the reactants are not uniformly distributed throughout the plasma. This condition may cause nonuniform reaction over the surface of the wafer. ${ }^{1}$ Nonuniform etching can have serious consequences in microelectronic fabrication in terms of reduced yield and quality of integrated circuits. Plasma etching reactors lack the necessary diagnostics for in situ monitoring of etch uniformity during processing. Optical emission from the plasma discharge may provide useful information on the spatial distribution of reactants in plasmas. We are exploring the use of optical imaging to derive 3-dimensional (3-D) spatial distributions of emitting species in plasmas and comparing their distributions with local etch rates of selected materials on patterned wafers.

The objectives of this research are (1) to develop optical diagnostics tor measuring the 3-D images of plasmas, (2) to n.easure the spatial distribution of reactants in a plasma reactor, and (3) to determine the efficacy of 3-D imagirg for in situ munitoring of etch uniformity during plasma processing of wafers.

This report describes an optical instrument for measuring and imaging low-level light emission from individual reactants in a glow discharge and an algorithm for transforming lateral optical emission signals to spatial maps of emitting species in the plasma. The plasma imaging system measures the spatial distribution of excited atomic chlorine at $726 \mathrm{~nm}$ in chlorinehelium plasmas during plasma etching of polysilicon, and the 3-D maps are compared with the measured radial etch rates of polysilicon on patterned silicon wafers. This plasma imaging system is recommended as an optical diagnostic for in situ measurement of etch uniformity on wafers.

\section{Experimental}

The plasma imaging system measures and maps the location and relative intensities of individual atomic, molecular, and ionized species in plasmas. Optical emission from the plasma discharge is measured through a grating monochromator, which separates incident light into component wavelengths. The imaging optics in the camera reconstructs an image of the plasma from monochromatic light to produce a 2-D map of a single emitting species in the plasma. The plasma image, when viewed and measured from the side, represents the integrated emission intensity over the entire thickness of the plasma. The lateral image is treated by Abel inversion to produce a 3-D map of emitting species in the plasma discharge.

The imaging camera, shown in Figure 1, consists of a $0.34 \mathrm{~m}$ Czerny-Turner monochromator with bilaterally adjustable entrance and exit slits, plane grating, single lens optics near the exit slit, image intensifier, video camera with video cassette recorder, and image processor, and is similar to the monochromatic imaging instrument described by Williamson, et al. ${ }^{2}$ The plasma discharge is viewed through a $1 \mathrm{~mm}$ entrance slit and focused onto a $1200 \mathrm{~g} / \mathrm{mm}$ grating (500 $\mathrm{nm}$ blaze) with a spherical mirror. The diffracted beam is directed onto a $1 \mathrm{~mm}$ exit slit with a second mirror in the Czerny-Turner mount. The spectral bandpass of this instrument is $2.4 \mathrm{~nm} / \mathrm{mm}$ in first order and covers the spectral recrion from 330 to 900 $\mathrm{nm}$. The monochromatic light, whose wavelength is determined by the angle of the diffraction grating in the monochromator, passes through the exit slit. The monochromatic image is focused on the image intensifier with a $100 \mathrm{~mm}$ focal length plano-convex lens (50 $\mathrm{mm}$ diameter) at a magnification of 0.05 . The intensified image is measured with a $C C D$ video camera, displayed on a video monitor, and recorded on a video cassette recorder at a standard video rate of 30 frames per second. The detector assembly consists of a Model IRO-01 intensified relay optics (Xybion Electronic Systems) and a Model 4815 video 
camera (Cohu, Inc.). The image processor, which is a Model FG-100-1024 single-board processor (Imaging Technology, Inc.) installed in an IBM-AT computer, analyzes real-time or recorded images by digitizing single frames or signal averaging up to 32 consecutive frames. The digitized plasma images are processed by Abel inversion to generate a spatial map of a single species in the plasma.

The plasma apparatus is a $45 \mathrm{~cm}$ cubic aluminum chamber with pumping from the side using a rotary mechanical pump. The plasma is generated in the center of the chamber by capacitive-coupling of a 13.56 $\mathrm{MHz}$ radio-frequency signal. The electrodes are configured asymmetrically with a lower, powered electrode mounted on a $200 \mathrm{~mm}$ diameter lucite base and an upper, $175 \mathrm{~mm}$ diameter grounded electrode. The electrode spacing was fixed at approximately $50 \mathrm{~mm}$ for the measurements reported here. The gas is delivered directly into the plasma through the grounded electrode in showerhead configuration. Mass-flow controllers are used to regulate the gas delivery rate. Radio-frequency power is coupled to the plasma through a $\mathrm{Pi}$ matching network with a blocking capacitor between the power supply and the network.
The powered electrode is allowed to float to a spontaneous self-bias potential. The potential relative to ground is monitored with an oscilloscope using a highvoltage probe attached to the powered electrode. The plasma is generated from a gas mixture of $60 \mathrm{sccm}$ helium and $30 \mathrm{sccm}$ chlorine with a total pressure of $125 \mathrm{~m}$ Torr and $140 \mathrm{~W}$ of radio-frequency power. A $150 \mathrm{~mm}$ diameter patterned silicon wafer is placed on the powered electrode. The base pressure of the plasma chamber is less than $1 \mathrm{~m}$ Torr. No provisions were made to control the temperature of the wafer platform during these experiments. Monochromatic images of the plasma were measured through an acrylic window mounted on the chamber at a sourceto-slit distance of $1400 \mathrm{~mm}$.

Patterned silicon wafers (150 mm diameter) were fabricated in the Microelectronics Development Laboratory at Sandia National Laboratories. The wafer has a layer of silicon dioxide $(305 \mathrm{~nm})$ over polysilicon $(1100 \mathrm{~nm})$. The layered structure was processed by photolithography and plasma etching to produce a highly patterned surface with exposed silicon dioxide, polysilicon, and photoresist. The line idths of exposed silicon dioxide and polysilicon vary from 100 to $400 \mu \mathrm{m}$. Etch uniformity and etch rates were determined by profilometry of the processed wafers.

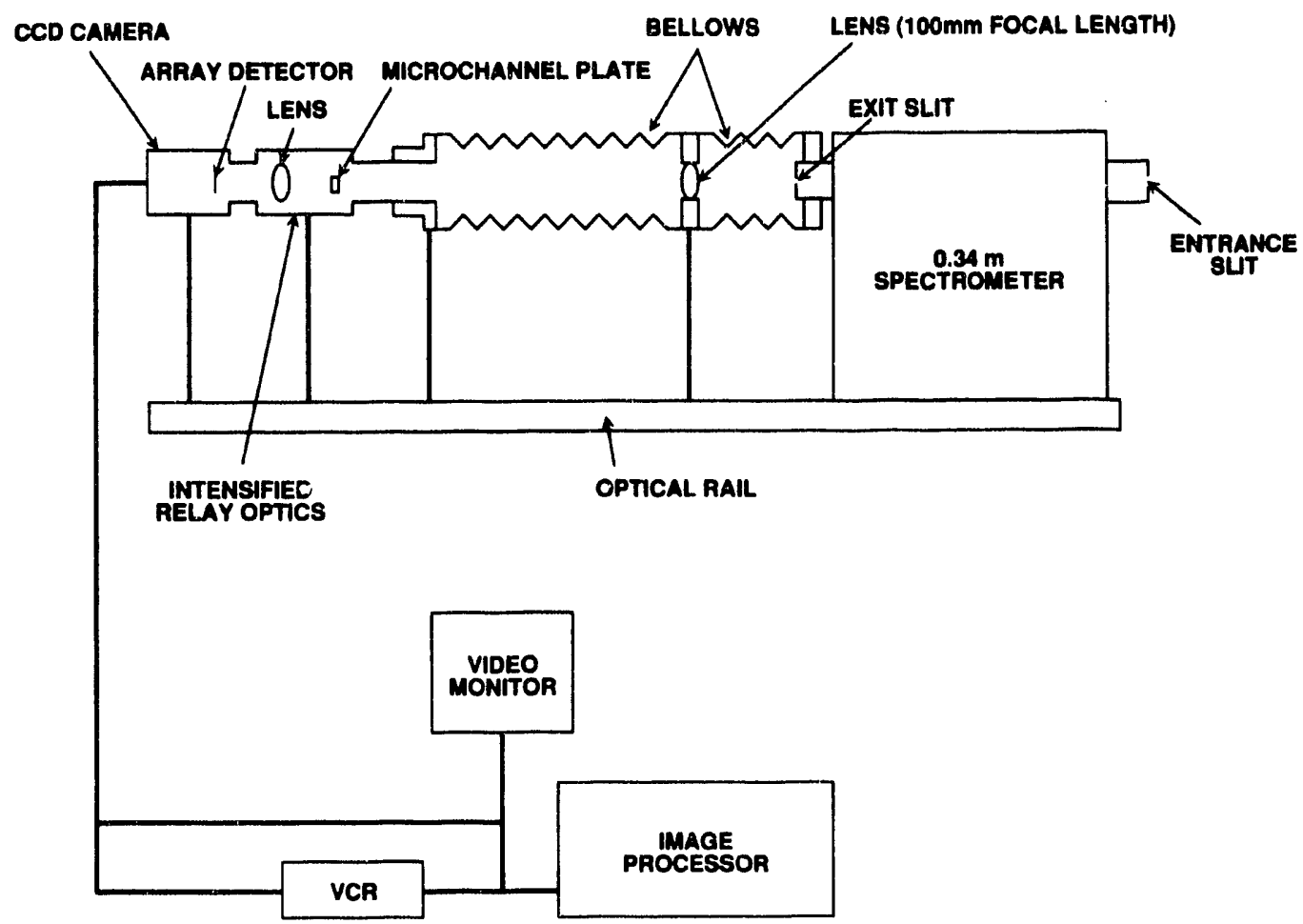

Figure 1. Imaging Camera for Measuring and Processing Monochromatic Video Images of Radio-Frequency Plasmas 
Abel inversion is based on the relationship between the lateral and radial emission intensities in axisymmetric sources. When measuring an optical emission signal at any position, y, along a horizontal plane in an axisymmetric plasma, the intensity at $y$ equals the integrated intensity over the entire thickness, $x$, of the plasma as shown in Figure 2. The relationship between the lateral intensity, $I(y)$, and radial intensity, $\mathrm{I}(\mathrm{r})$, is given by the following equation:

$$
I(y)=\int_{-x}^{+x} I(r) d x
$$

By simple substitution, the following relationship can be derived:

$$
I(y)=\frac{2}{y} \int^{R} r I(r)\left(r^{2}-y^{2}\right)^{-1 / 2} d r
$$

If the plasma source is axisymmetric, $\mathrm{I}(\mathrm{r})$ can be determined by the inverse formula, which is Abel's integral equation: ${ }^{3}$

$$
I(r)=-1 / \pi \int_{r}^{R} I^{\prime}(y)\left(y^{2}-r^{2}\right)^{-1 / 2} d y
$$

The lateral intensity profile is measured at a particular observation height, $h$, in the cylindrical plasma and fit to a polynomial using least squares analysis. The first derivative of the polynomial, $I^{\prime}(y)$, is used in the integral to calculate the radial mission intensity, $I(r)$, from the center to the right edge of the plasma. This process is repeated for the left half to produce a radial intersity profile at each observation height in the plasma. The discontinuity at $y=r$ presents computational problems that must be treated separately in the calculations. Integration of Equation (3) has been described in detail. ${ }^{3}$

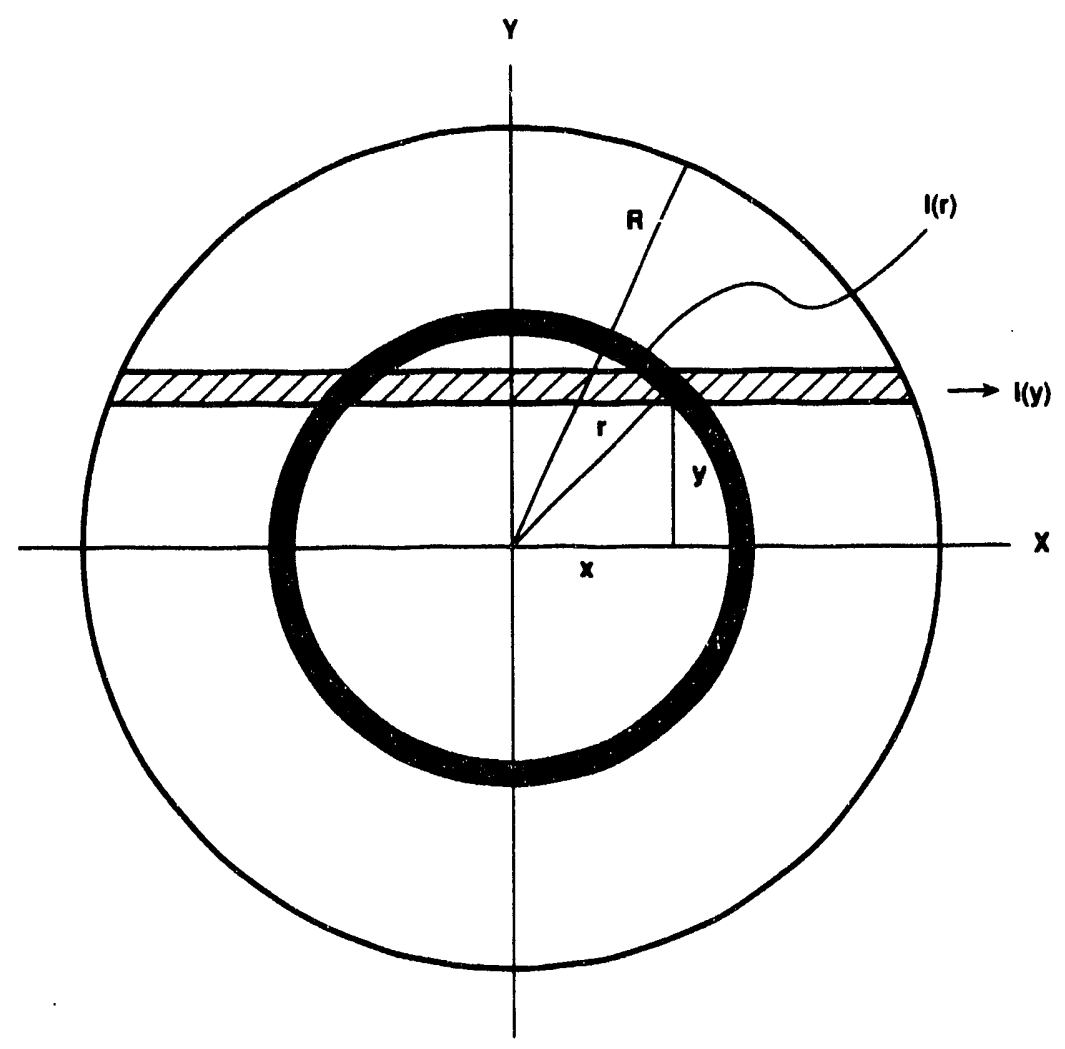

Figure 2. Diagram showing the Relationship Between the Lateral Emission Intensity, I(y), and Radial Emission Intensity, I(r), Through a Horizontal Plane in an Axisymmetric Plasma 


\section{Results and Discussion}

The purpose of this research is to investigate optical imaging techniques for monitoring etch unifornity in plasmas. Optical spectra of plasmas can be used for distinguishing and identifying excited atomic, molecular, and ionized species. By imaging the emission signal of the plasma discharge at a particular wavelength, one can determine the spatial distribution and relative concentration of a single species. Such information would be extremely useful in a variety of ways. If the reactant species plays a key role in chemical etching, as measured by comparing the local etch rate with the corresponding emission intensity, one could estimate the degree of etch uniformity over the wafer surface and monitor, in real time, etch uniformity in the plasma. Conversely, if there is a poor correlation between local etch rate and emission intensity for a particular species, etch rate would be independent of the species concentration in the plasma.

We investigated nonuniform etching reactions in chlorine-helium plasmas and applied plasma imaging techniques to the in situ measurement of etch uniformity. Figure 3 is a photograph of a $150 \mathrm{~mm}$ diameter silicon wafer with exposed polysilicon, silicon dioxide, and photoresist. The unprocessed wafer has a uniform appearance. After processing the wafer in a chlorinehelium plasma for 20 minutes and post-plasma treatment in an oxygen plasma, we observed a characteristic "bulls-eye," or concentric ring pattern, that is due to constructive interference of reflected light on the wafer, as shown in Figure 4. Profilometry measurements of the wafer showed that polysilicon was etched to a much greater extent near the edge than at the center. The measured etch rate, in nanometers per minute $(\mathrm{nm} / \mathrm{min})$, is plotted as a function of the radial position on the wafer, and the results are shown in Figure 5 . The etch rate varies from $34.5 \mathrm{~nm} / \mathrm{min}$ at the center to $41.5 \mathrm{~nm} / \mathrm{min}$ near the edge. The results show that nonuniform etching does indeed occur in this plasma under the plasma conditions described in the Experimental section.

The optical emission spectrum of the chlorinehelium plasma, which is shown in Figure 6, shows characteristic spectra of atomic chlorine and helium and molecular chlorine species. The most intense helium emissions were found at $388.9 \mathrm{~nm}$ and 501.6 $\mathrm{nm}$. The small peak at $656.3 \mathrm{~nm}$ is probably atomic hydrogen. Broadband emission in the wavelength region from 400 to $600 \mathrm{~nm}$ corresponds to molecular chlorine species in the plasma. ${ }^{4}$

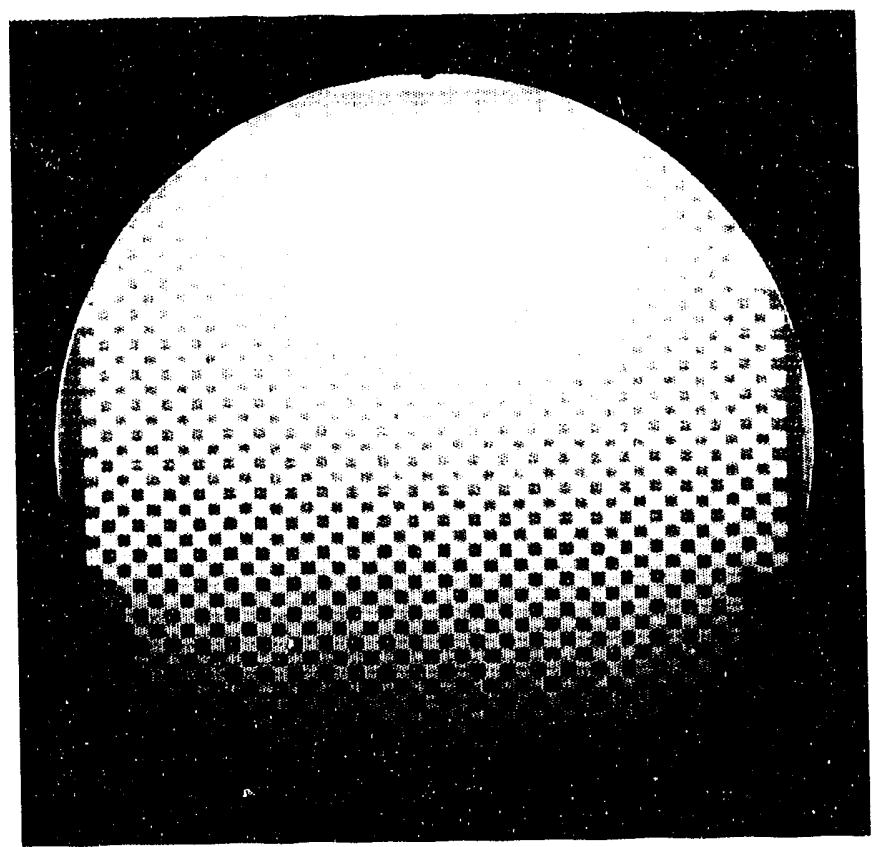

Figure 3. Patterned Silicon Wafer $(150 \mathrm{~mm}$ diameter) with Exposed Silicon Dioxide, Polysilicon, and Photoresist

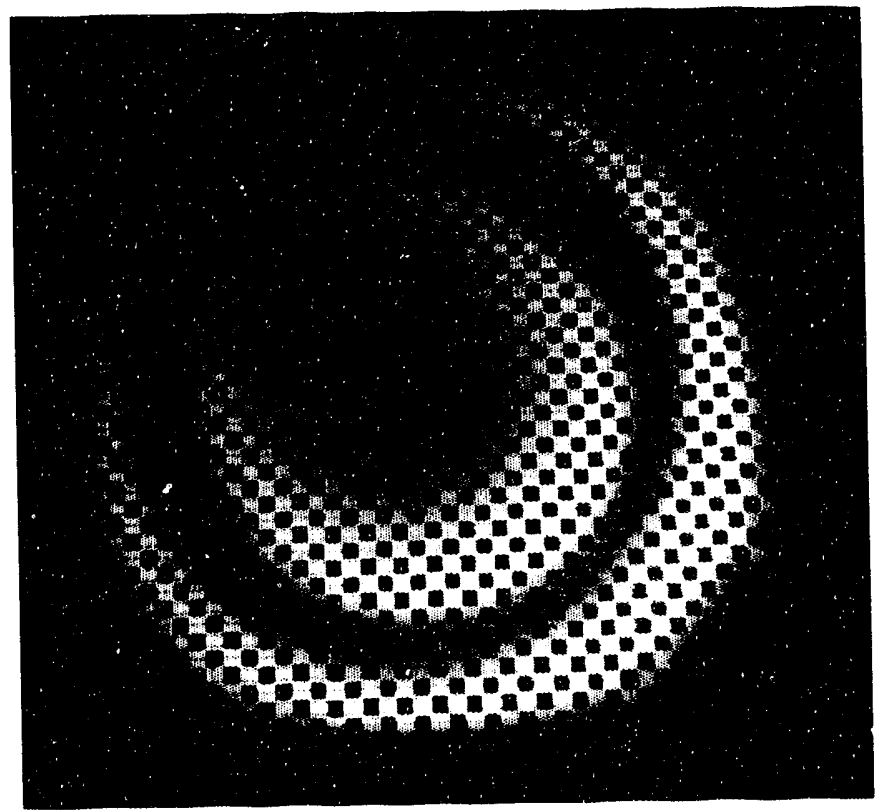

Figure 4. Silicon Wafer in Figure 3 after Etching in a Chlorine-Helium Plasma and Post-Plasma Treatment in an Oxygen Plasma 


\section{Etch Rate of Polysilicon}

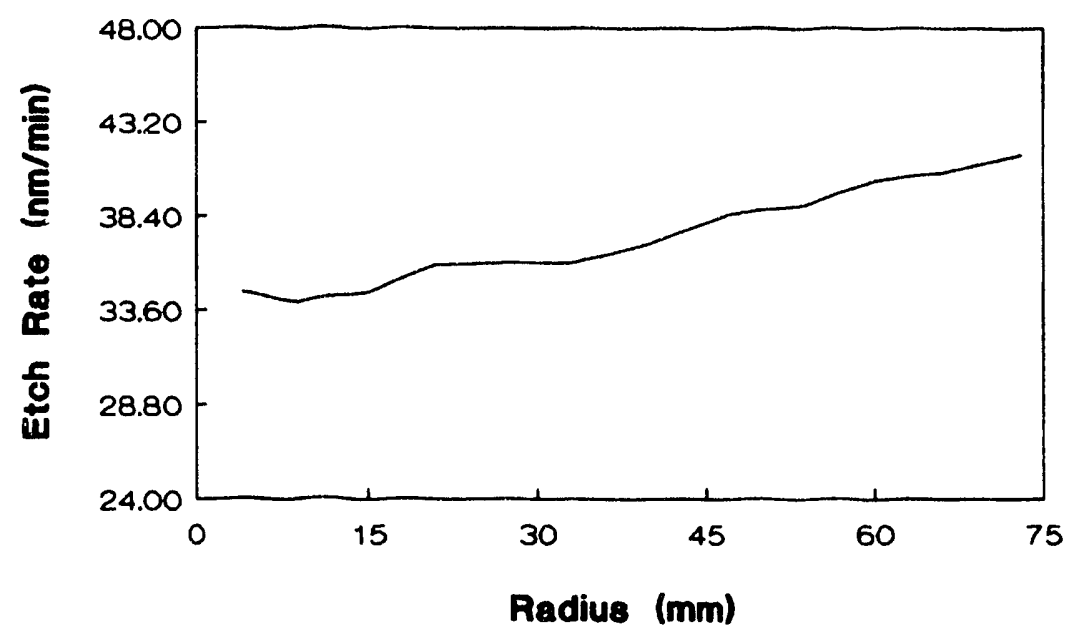

Figure 5. Etch Rate of Polysilicon as a Function of Radial Position on the Plasma-Processed Wafer

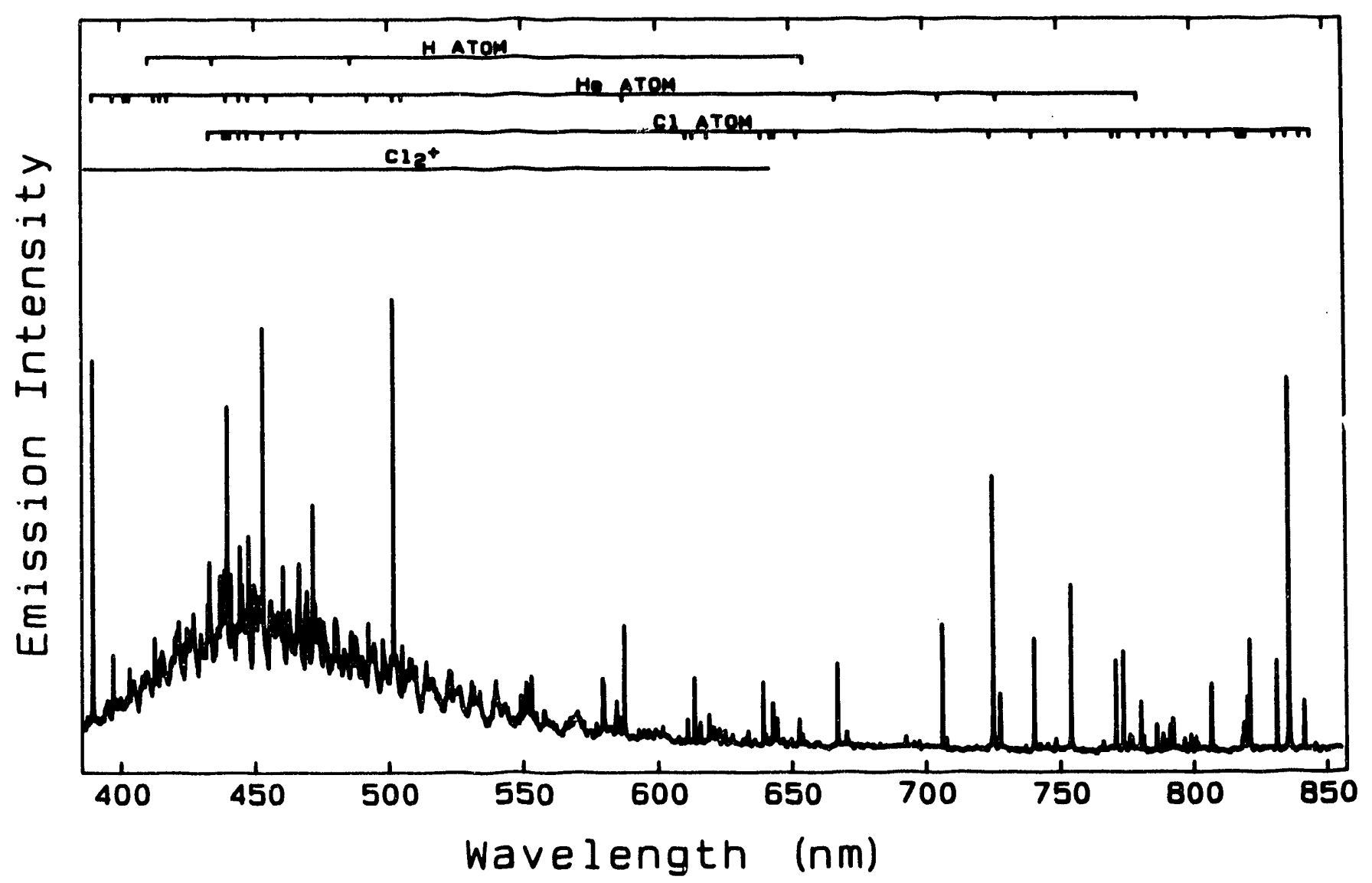

Figure 6. Emission Spectrum of Chlorine-Helium Plasma 
Intense atomic chlorine emission is observed at $725.7 \mathrm{~nm}$, and the peak is resolved sufficiently to permit imaging of excited atomic chlorine without interference from other species in the plasma. Figure 7 is a monochromatic image of a chlorine-helium plasma at $725.7 \mathrm{~nm}$. The side-on view shows intense emission between the parallel electrodes with a small secondary plasma below the wafer platform. The colors in the computer-enhanced images represent a graduated intensity scale that ranges from blue to red. The radial emission intensity of atomic chlorine was determined by Abel inversion of the image in Figure 7. The transformed image in Figure 8 reveals a structured plasma in which atomic chlorine emission varies both axially and radially in the discharge. Chlorine atomic emission is most intense near the edge of the plasma discharge and decreases gradually toward the center.

The intensity profile for atomic chlorine was measured in a volume of the plasma that extended $5 \mathrm{~mm}$ above the wafer surface by ensemble-averaging consecutive horizontal segments in the transformed image. This region of the plasma would be representative of the plasma environment to which the wafer is exposed since the mean-free-path of atomic chlorine is on the order of $1 \mathrm{~mm}$ at an operating pressure of 125 mTorr. Figure 9 shows the emission intensity of atomic chlorine as a function of the radial position above the wafer surface in a $5 \mathrm{~mm}$ high segment of the plasma. The graph shows a relatively low atomic chlorine emission intensity near the center and a higher atomic chlorine emission intensity near the edge of the wafer, which is similar to the calculated radial etch rates in Figure 5. Based on these experimental results, the correlation appears strong between the atomic chlorine emission intensity in a volume of the plasma gas directly above the wafer surface and the local etch rate on the silicon wafer surface.

\section{Summary}

The plasma imaging system described in this report produces high-resolution images from single excited species in radio-frequency plasmas. The instrument has high spectral, spatial, and temporal resolution and is extremely sensitive for measuring low-level light emission from reactive gas plasmas. Radial distributions of individual excited species in plasmas are determined by Abel inversion. Monochromatic images of atomic chlorine in chlorine-helium plasmas were measured at $725.7 \mathrm{~nm}$. Radial images of these plasmas showed that the intensity of excited atomic chlorine varies radially across the wafer surface, with a minimum near the center and maximum near the periphery of the wafer. The polysilicon etch rate varies fiom $34.5 \mathrm{~nm} / \mathrm{min}$ at the center to 41.5 $\mathrm{nm} / \mathrm{min}$ near the edge of the silicon wafer. There is a direct correlation between the radial emission intensity of atomic chlorine and the etch rate for polysilicon. If we can establish a direct correlation between the emission intensities for a number of individual reactive species in plasmas and the etch rates for selected materials on wafers, the plasma imaging instiunent will be a valuable diagnostic tool for in situ inonitoring of etch uniformity in plasmas. In addition, this technique may be an important research tool for studying gas-solid reactions in low-temperature plasmas.

\section{References}

${ }^{1}$ R. C. Booth and C. J. Heslop, Application of Plasma Etching Techniques to Metal-Oxide-Semiconductor (MOS) Processing, Thin Solid Films, vol 65, 111-23 (1980).

${ }^{2}$ R. L. Williamson, W. A. Hareland, and H. C. Peebles, Simple Compact Monochromatic Imaging System for Plasma Diagnostics, Appl. Spect., vol 28, no. 12, 2201-03 (1989).

${ }^{3} \mathrm{C}$. Fleurier and J. Chapelle, Inversion of Abel's Integral Equation - Application to Plasma Spectroscopy, Computer Physics Communication, vol 7, 200-06 (1974).

${ }^{4} \mathrm{R}$. W. B. Pearse and A. G. Gaydon, The Identification of Molecular Spectra, John Wiley \& Sons Inc., New York, 1950. 


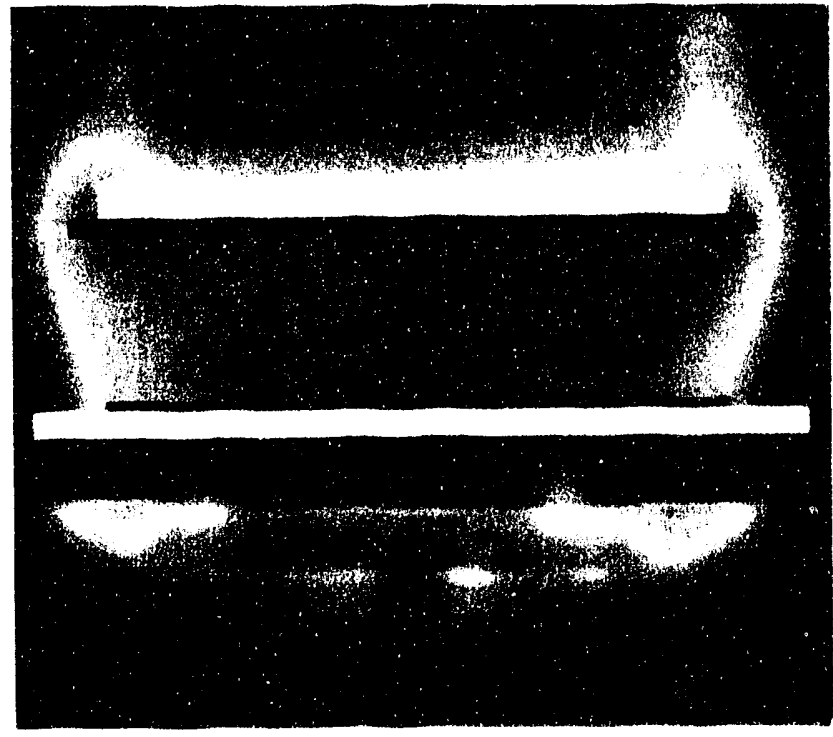

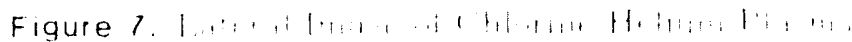

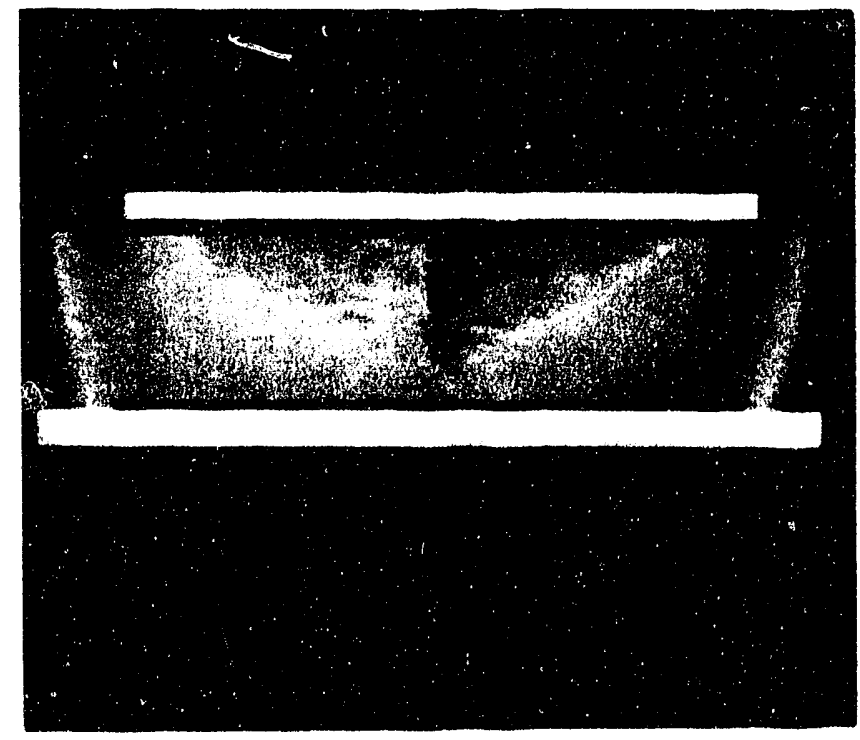

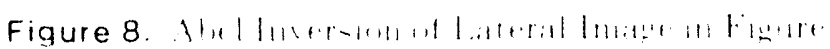

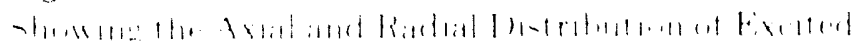

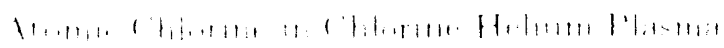
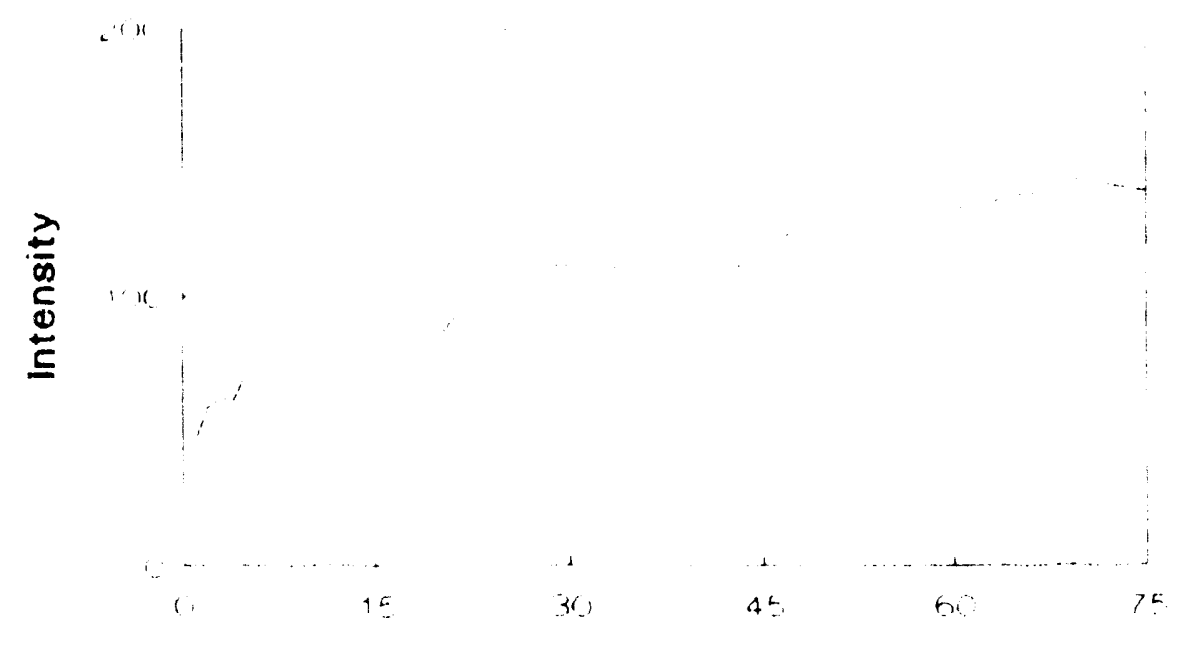

Radius $(\mathrm{mm})$

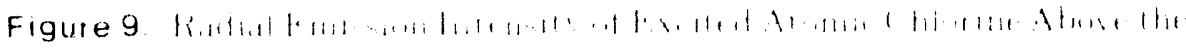

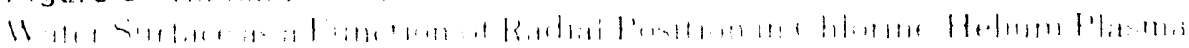




\section{DISTRIBUTION:}

$\begin{array}{rll}3 & 251 & \text { Intellectual Property Dev Dept } \\ 1 & 1111 & \text { J. A. Knapp } \\ 1 & 1128 & \text { K. E. Greenberg } \\ 1 & 1128 & \text { P. J. Hargis, Jr } \\ 1 & 1128 & \text { P. A. Miller } \\ 1 & 1128 & \text { M. E. Riley } \\ 5 & 1302 & \text { C. W. Gwyn } \\ 5 & 1305 & \text { R. S. Blewer } \\ 1 & 1324 & \text { D. A. Brown } \\ 1 & 1324 & \text { B. K. Smith } \\ 1 & 1324 & \text { H. D. Stewart } \\ 1 & 1325 & \text { M. G. Blain } \\ 1 & 1325 & \text { P. J. Resnick } \\ 1 & 1512 & \text { D. J. Rader } \\ 10 & 1812 & \text { R. J. Buss } \\ 5 & 1812 & \text { C. L. Renschler } \\ 1 & 1824 & \text { R. P. Goehner } \\ 10 & 1824 & \text { W. A. Hareland } \\ 5 & 1824 & \text { M. R. Keenan } \\ 1 & 1841 & \text { R. A. Neiser, Jr } \\ 1 & 1841 & \text { M. F. Srnith } \\ 1 & 4200 & \text { T. M. Dyer, Actg } \\ 1 & 4212 & \text { R. A. Hamil } \\ 5 & 6612 & \text { N. E. Brown } \\ 1 & 8351 & \text { G. A. Fisk, Actg } \\ 1 & 8353 & \text { F. P. Tully } \\ 1 & 8361 & \text { D. R. Hardesty } \\ 1 & 8362 & \text { R. W. Carling } \\ 1 & 8713 & \text { D. K. Ottesen } \\ 1 & 8523-2 & \text { Central Technical Files } \\ 5 & 7141 & \text { Technical Library } \\ 1 & 7151 & \text { Technical Publications } \\ 10 & 7613-2 & \text { Document Processing } \\ & & \text { For DOE/OSTI } \\ & & \\ 1 & \end{array}$



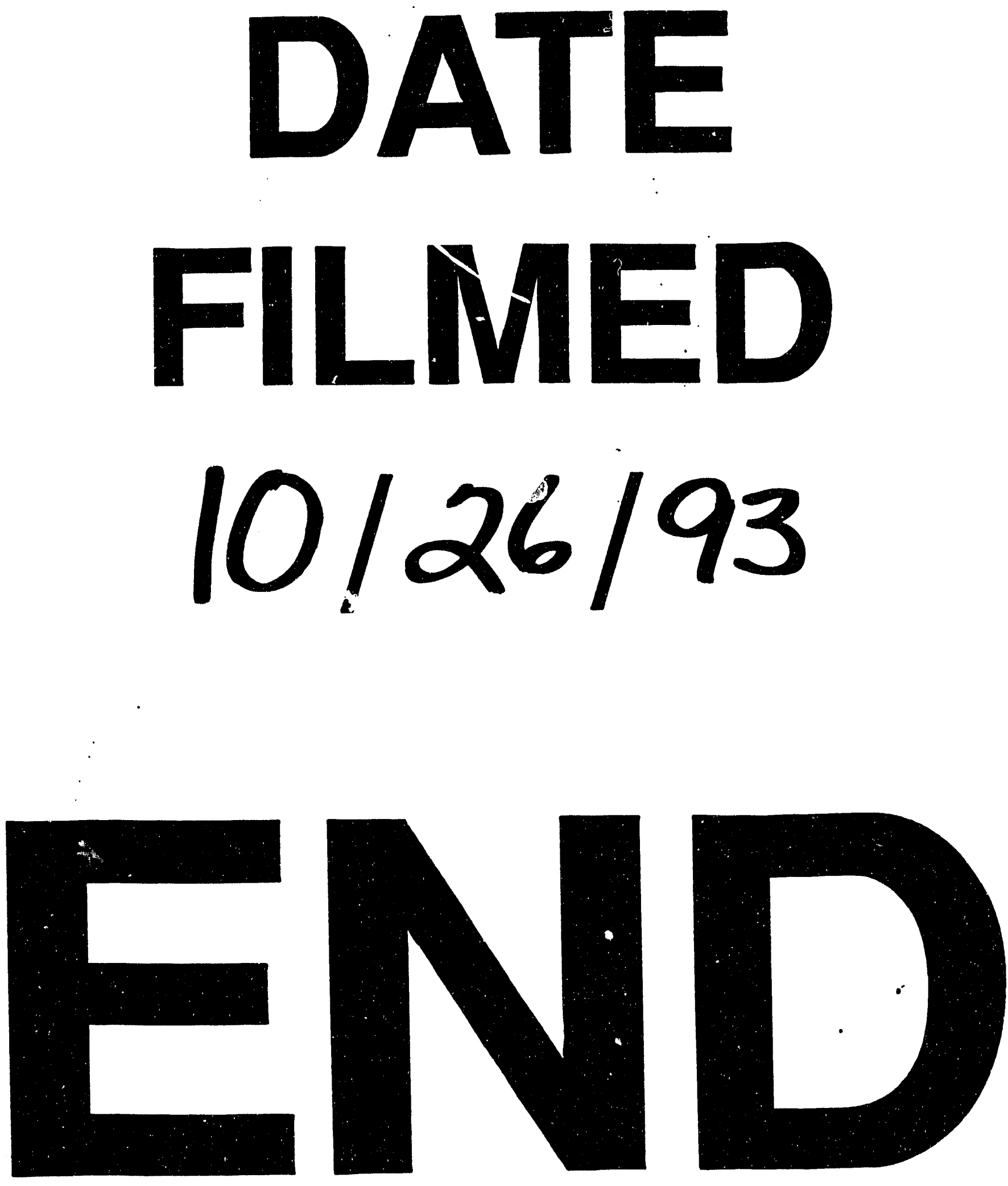
